\title{
Esteróide anabolizante inibe a angiogênese induzida pelo treinamento físico de natação em músculo sóleo de ratos normotensos
}

CDD. 20.ed. 796.022

796.073

797.2

\author{
Ursula Paula Reno SOCI* \\ Fernanda Roberta Roque REDONDO* \\ Tiago FERNANDES ${ }^{*}$ \\ Kátia De ANGELIS ${ }^{* * *}$ \\ Maria Cláudia IRIGOYEN** \\ Marcele COELHO" \\ Edilamar Menezes de OLIVEIRA*
}

*Escola de Educação

Física e Esporte,

Universidade de São

Paulo.

**Instituto do Coração, Hospital das Clínicas, Faculdade de Medici-

na, Universidade de

São Paulo.

***Universidade São Judas Tadeu.

\section{Resumo}

Os esteróides anabolizantes androgênicos (EAA) são sintéticos de testosterona desenvolvidos para fins terapêuticos. São também utilizados por populações fisicamente ativas, que normalmente excedem nas doses, o que potencializa danos à saúde. Para estudar alguns dos efeitos de EAA sobre o sistema cardiovascular, ratos "Wistar" foram divididos em quatro grupos: sedentário controle (SC), sedentário anabolizado (SA), treinado controle (TC) e treinado anabolizado (TA). Foram avaliados os efeitos da associação do uso de EAA (Decanoato de nandrolona - $5 \mathrm{mg} / \mathrm{kg} \mathrm{sc}, 2 x / \mathrm{sem}$ ) e do treinamento fisico de natação (TFN - $60 \mathrm{~min} / \mathrm{dia}, 5 \mathrm{x}$ ) sem, durante $10 \mathrm{sem}$ ) sobre o débito cardíaco (DC) e fluxo sanguíneo basal (DCbasal, Qbasal) e após infusão do vasodilatador acetilcolina (DC Ach, $Q$ Ach) para observar a vasodilatação endotélio dependente (QAch), razão capilar/fibra (rc/f) e expressão do fator de crescimento endotelial vascular (VEGF) em músculo sóleo (predominância de fibras oxidativas). A testosterona plasmática aumentou nos grupos com uso de EAA e foi observada bradicardia de repouso como efeito do TFN. O DC foi menor para o Grupo TA, tanto na condição basal quanto sob infusão de Ach. 0 Qbasal não foi diferente entre os grupos no músculo estudado. 0 QAch foi maior no grupo TC, entretanto, no grupo TA este efeito benéfico do TFN foi prejudicado pela associação com o EAA. Aumento da rc/f e VEGF foi observado somente no grupo TC. Estes resultados sugerem que a associação do EAA ao TFN atenua a angiogênese e arteriogênese observadas como efeito do treinamento físico aeróbio e causa prejuízo ao fluxo sanguíneo muscular, o que poderia predispor o praticante de esportes e atividades físicas e usuário destas substâncias a problemas vasculares.

Unitermos: Doping; Fluxo sanguíneo; Capilares; Músculo esquelético; Treinamento físico; Natação.

\section{Introdução}

Os esteróides anabolizantes androgênicos (EAA) são sintéticos com atividade similar à testosterona, utilizados para fins terapêuticos e no meio esportivo devido à suas propriedades anabólicas e androgênicas (Celotti \& Cesi, 1992; Hebert. Haupt, George \& Rovere, 1984; Kuhn, 2002). Estas drogas são frequentemente utilizadas em academias e centros de treinamento físico sem qualquer critério e controle, o que representa risco à saúde dos usuários (Andersen, Bartlett, Morgan \& Brownell, 1995; Fuller, 1993). As doses costumam ser de 10 a 100 vezes maiores que a terapêutica, e $2 / 3$ dos abusos ocorrem entre não atletas (Cunha, Moura, Bernardes, Tanno \& Marcondes, 2005; Kam \& Yarrow, 2005; Pope JUNIOR \& KATZ, 1988). 
O uso abusivo de EAA causa diversos efeitos deletérios para o organismo, com consequências adversas de ordem metabólica, endócrina, cardiovascular, hepática, neural, estética, comportamental e psiquiátrica (KINDERMANN, 2006; ManetTa \& Silveira, 2000; Redondo, 2007; Rocha, Carmo, Roque, Hashimoto, Rossoni, Frimm, Anéas, Negrão, Krieger \& Oliveira, 2007; Tagarakis, Bloch, Hartmann, Hollmann \& Addicks, 2000; TAKAHASH, TATSUGi \& KoHnO, 2004).

As adaptações funcionais e morfológicas da musculatura esquelética e outros sistemas decorrentes de treinamento físico de natação (TFN) são bem descritas, resultando em modificações metabólicas (capacidade oxidativa muscular e otimização nos sistemas de produção de energia) e circulatórias centrais e periféricas (débito cardíaco, fluxo sanguíneo muscular, aumento de capilares) (Costill, Maglischo \& Richardson, 1992). Destas, no músculo esquelético, considera-se a angiogênese uma importante adaptação decorrente do treinamento de resistência aeróbia. $\mathrm{O}$ aumento no número de capilares ao redor da fibra pode ser até $50 \%$ maior nos músculos de membros superiores de nadadores em relação aos sedentários, o que permite uma melhor permuta de gases, calor e substratos energéticos entre o sangue e fibra muscular ativa (Costill, Maglischo \& RichARDSON, 1992; INGJER \& BRODAL, 1978; SALTIN $\&$ Rowell, 1980). Esta adaptação é deflagrada fisiologicamente por poucas situações como o ciclo ovariano, desenvolvimento placentário e em resposta ao aumento do estresse de cisalhamento e ao estiramento mecânico celular no músculo esquelético que recebe sobrecargas como a estimulação elétrica e o exercício físico (PRIOR, YANG \& Terjung, 2004).

O TFN, bem como outros tipos de treinamento de resistência aeróbia, é capaz de induzir a um aumento na proliferação de células endoteliais em fibras do tipo I e II no músculo esquelético, mostrando a expressão aumentada de compostos angiogênicos, como o VEGF (Vascular-Endothelial Growth Factor) no músculo e circulação (JENSEN, Bangsbo \& Hellsten, 2004; Kraus, Stallings, Yeager \& Gavin, 2004; Waters, Rotevatin, Ping, Anexx \& Yan, 2004). Este fator de crescimento é específico para a microvasculatura, sendo um potente mitógeno de células endoteliais relacionado ao controle da formação de novos capilares, e o principal implicado na resposta angiogênica ao exercício (Amaral, Papanek \& Greene, 2001;
Bornes, Boulard, Hieblot, Zanibellato, Iacovoni, Prats \& Touriol, 2004; Kraus, Stallings, Yeager \& GaVin, 2004).

VEGF e seus receptores atualmente são considerados elementos críticos para a ocorrência da angiogênese "in vivo". A interação entre o receptor VEGFR2 e VEGF estimula a proliferação e migração de células endoteliais, através de uma cascata de sinalização Tirosina quinase, que ativa consequentemente Fosfolipase-C- $\gamma$ (PLC-C $\gamma$, que catalisa hidrólise de ligações éster em fosfolipídeos), Fosfoinositol-3-quinase (PI3K, enzima que fosforila inositol e que participa de múltiplos papéis na ativação celular) e proteína quinase $\mathrm{C}-€$ (PKC-€ , enzima que está envolvida também em muitos eventos de transdução de sinais, sendo ativa em resposta a estímulos hormonais, neuronais e como neste caso, de fatores de crescimento e catalisando a transferência de um grupo fosfato do ATP a diversas proteínas substrato) que estimulam expressão de genes, incluindo de eNOS (sintetase catalisadora da produção de Oxido Nítrico endotelial - NO) cuja atividade é crítica para a sinalização do VEGF (Amaral, Papanek \& Greene, 2001; Bornes et al., 2003; Kraus et al., 2004; Prior, Yang \& Terjung, 2004, WATERS et al., 2004).

Por outro lado, é bem estabelecido que a associação de dose suprafisiológica de EAA com treinamento físico causa efeitos deletérios para diversos sistemas, dentre eles o cardiovascular, resultando em diminuição da capilarização no músculo cardíaco e esquelético de animais (Redondo, 2007; Soares \& Duarte, 1991; TAGARAKIs et al., 2000), porém não se sabe como os compostos angiogênicos, como o VEGF, se comportam nesta associação.

Embora se conheça vários efeitos benéficos do treinamento físico de resistência aeróbia em relação ao músculo esquelético, pouco se sabe sobre sua associação com o uso de EAA. A maioria dos estudos relaciona o uso destes recursos a atletas de força ou que treinam em alta intensidade. Pouco foi elucidado sobre os efeitos da associação do TFN e de doses suprafisiológicas de EAA na angiogênese no músculo esquelético. Desta forma, o presente estudo teve como objetivo avaliar os efeitos de dose suprafisiológica de EAA sobre o remodelamento da microvasculatura, expressão protéica do VEGF e fluxo sanguíneo no músculo sóleo, o músculo com maior potencial de adaptabilidade ao treinamento de resistência aeróbia, verificando se a droga é capaz de alterar a angiogênese induzida pelo TFN. 


\section{Materiais e métodos}

\section{Grupos experimentais}

Ratos "Wistar" (8-12 semanas, 180-250 g, $\mathrm{n}=56$ ), foram separados em quatro grupos randômicos conforme o protocolo experimental: Sedentário Controle (SC, $\mathrm{n}=14$ ), Sedentário Anabolizado (SA, $\mathrm{n}=14$ ), Treinado Controle (TC, $\mathrm{n}=14)$ e Treinado Anabolizado (TA, $n=14$ ), identificados, mantidos com ração e água "ad libitum", em gaiolas plásticas a temperatura controlada $\left(23 \pm 1^{\circ} \mathrm{C}\right)$ e ciclo claro-escuro invertido a cada 12:12 h durante o experimento. Foi realizado controle ponderal dos animais semanalmente. Cada grupo foi subdividido em dois grupos, um utilizado para análises bioquímicas, moleculares, morfológicas e morfométricas e outro para análises hemodinâmicas e de perfusão regional.

Os grupos SA e TA foram tratados com Decanoato de Nandrolona (Decadurabolin; Organon, Roseland, NJ) administrado por injeções subcutâneas duas vezes por semana, totalizando 10 $\mathrm{mg} \mathrm{kg-1/semana.} \mathrm{Esta} \mathrm{dosagem} \mathrm{representa} \mathrm{aproxi-}$ madamente 100 vezes a dosagem terapêutica (PoPE JUNIOR \& KATZ, 1988). As injeçóes foram preparadas com diluição em óleo vegetal e aplicadas sempre no período da tarde. Os grupos que não foram tratados com a droga (SC e TC) receberam a administração de óleo vegetal.

Todos os procedimentos cirúrgicos e protocolos foram realizados de acordo com os Princípios Éticos de Experimentação Animal (ColÉGIO Brasileiro de Experimentação Animal - COBEA, 1991).

\section{Treinamento físico de natação}

O TFN (TC e TA) foi realizado conforme descrito por Medeiros, Oliveira, Gianolla, Casarini, Negrão e Brum (2004) (FIGURA 1), com frequência de cinco vezes por semana em um sistema com água aquecida entre $30-32^{\circ} \mathrm{C}$, duração de $60 \mathrm{~min} /$ dia por 10 semanas com sobrecarga de trabalho (peso adaptado à cauda) de $5 \%$ do peso corporal (FIGURA 1). Este é um protocolo de baixa intensidade e longa duração, efetivo para a promoção de adaptaçôes cardiovasculares e aumento na capacidade oxidativa muscular-esquelética (Medeiros et al., 2004).

Para o estudo de perfusão regional, 24 horas após o final do protocolo experimental os animais foram submetidos ao processo cirúrgico para realização do experimento com as microesferas coloridas, o qual foi realizado 24 horas após o procedimento cirúrgico, seguido do sacrifício dos animais e coletas.

Para realização de análises morfológicas, morfométricas, bioquímicas e moleculares, o outro grupo foi sacrificado 24 horas após o final do protocolo experimental e o músculo sóleo foi retirado e adequadamente armazenado para as diferentes análises.

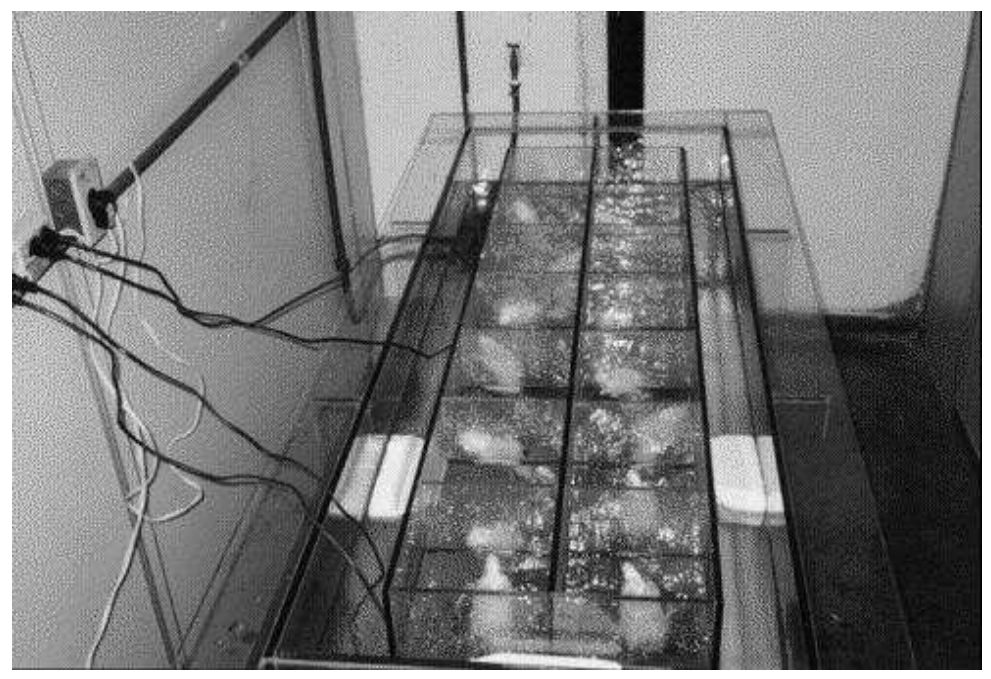

FIGURA 1 - Sistema aquecido de natação para ratos. 
Análises morfológicas e morfoméricas

Análise histológica

Quantificação do número de capilares

Ao final do protocolo experimental os animais foram sacrificados e seus músculos sóleos foram removidos e pesados. Os músculos foram seccionados transversalmente, fixados em formaldeído a $6 \%$ por 24 horas e processados por desidratação, diafanização e banho de parafina a $60{ }^{\circ} \mathrm{C}$. Após foi realizada a inclusão dos mesmos em cassetes contendo parafina fundida, que ao solidificar deu origem a um bloco de parafina para cada sujeito da amostra, dos quais se obteve cortes microtômicos de $3 \mu \mathrm{m}$ de espessura, submetidos à montagem de lâminas para análise histológica, com coloração de PAS (Periodic Acid Schiff), para visualização dos vasos capilares.

A análise histológica foi realizada com utilização do sistema de análise de imagens Quantimet Leica ${ }^{\circledR}$, (Leica, Cambridge, United Kingdom) por intermédio de estudo cego. A lâmina foi observada inicialmente em aumentos menores, sendo escolhido um local em que não havia ranhuras ou bolhas e que contenham o maior número de fibras de corte transversal. Escolhido o local, a imagem foi ampliada 400x para realizar as medidas. Para análise do número de capilares, a mesma área foi igualmente delimitada, com o auxílio do cursor para mensurar o diâmetro de cada vaso. Segundo critérios estabelecidos, o diâmetro foi o principal parâmetro para identificação dos capilares, sendo considerado capilar, vasos com diâmetro igual ou menor que $12 \mu \mathrm{m}$.

Foram analisados cinco campos de visão das lâminas de cada amostra nas quais foi quantificado o número total de fibras musculares e de capilares por campo de visão e a partir destes valores foi calculada a razão Capilar/Fibra (rc/f) de cada animal, como preditor de capilarização no tecido estudado.

\section{Hipertrofia muscular esquelética}

A hipertrofia foi avaliada através da relação entre o peso de cada músculo (peso úmido) e o peso corporal. Os resultados são expressos como razão peso do sóleo/peso corporal do animal (mg/g).

\section{Fluxo sanguíneo muscular Técnica das microesferas coloridas}

O fluxo sanguíneo para o músculo sóleo foi avaliado pela técnica de microesferas coloridas como previamente descrito por HaKkinen, Miller, SMITH e KNIGHT (1995). Vinte e quatro horas após a última sessão de exercício os ratos foram anestesiados com ketamina $(90 \mathrm{mg} / \mathrm{kg})$ e xilasina $(10 \mathrm{mg} / \mathrm{kg}$, ip). Cateteres preenchidos com salina foram implantados na artéria femoral para a retirada de uma amostra de sangue referência e dentro do ventrículo esquerdo para infusão das microesferas coloridas. Uma terceira cânula foi introduzida na veia femoral direita, para administração do vasodilatador acetilcolina (Ach).

Durante o experimento, que foi realizado 24 horas após o processo cirúrgico, os animais permaneceram conscientes e foi permitido que se movessem livremente. A cânula da artéria femoral foi conectada a um transdutor de pressão e os sinais de pressão arterial foram continuamente monitorados, exceto durante a infusão de microesferas e a retirada das amostras de sangue referência. Os dados registrados foram analisados batimento a batimento para quantificar mudanças na pressão arterial média e frequência cardíaca.

Durante o repouso microesferas coloridas vermelhas (200.000) foram infundidas e utilizadas para a determinação do fluxo sanguíneo muscular esquelético e débito cardíaco. Em uma segunda etapa o procedimento foi repetido com microesferas na cor branca, porém antecedido pela administração de um vasodilatador (acetilcolina - Ach) na dose de $30 \mu \mathrm{g} /$ $\mathrm{kg}$, através da cânula localizada na veia femoral direita. Esta etapa foi realizada para avaliar a resposta vasodilatadora endotélio-dependente dos animais.

Após este procedimento os animais foram sacrificados e os músculos sóleos foram retirados para a determinação do fluxo sanguíneo. As amostras foram digeridas e processadas com reagentes preparados segundo especificaçōes do fabricante das microesferas (Triton Technology, San Diego, CA, USA). A infusão de microesferas e processamento das amostras de sangue e dos tecidos foram realizados pelo método de HAKKINEN et al. (1995). A absorbância mínima aceitável foi de 0,010 unidades de absorbância (AU). Para cada infusão os valores de fluxo foram representados como número de microesferas dentro do tecido e/ou como $\mathrm{ml} / \mathrm{kg}^{-1} / \mathrm{min}^{-1}$. O fluxo sanguíneo $\left(\mathrm{ml} / \mathrm{kg}^{-1} / \mathrm{min}^{-1}\right)$ e o débito cardíaco foram calculados como previamente descrito (HAKKINEN et al., 1995).

O número de microesferas nos músculos estudados foi normalizado pelo respectivo peso muscular. Para determinação do número de microesferas nos tecidos (brancas ou vermelhas) soluções com número conhecido de microesferas foram processadas. A média da absorbância destas 
amostras permite determinar uma constante de leitura do espectofotômetro para as microesferas vermelhas e brancas. Desta forma o número de microesferas nos tecidos foi calculado a partir de fórmulas previamente especificadas. O tempo de retirada da amostra de sangue $(\mathrm{min})$ foi calculado pela divisão do volume de sangue retirado durante a infusão das microesferas vermelhas e brancas, pela velocidade da bomba de retirada $(0,5 \mathrm{ml} / \mathrm{min})$. A constante de retirada do sangue ( $Q$ sangue, $\mathrm{ml} / \mathrm{min}$ ) foi determinada pela divisão do volume de sangue $(\mathrm{ml})$ pelo tempo de retirada da amostra (HAKKINEN et al., 1995).

\section{Análises bioquímicas e moleculares}

\section{Testosterona plasmática}

A concentração de testosterona plasmática foi determinada por Radioimunoensaio (COAT-ACOUNT total Testosterone da Diagnostic Products Corporation - humano) em plasma coletado com EDTA (ácido etilendinitrolotetracético). Os resultados foram expressos em $\mathrm{ng} / \mathrm{dl}$ de testosterona.

\section{Análise da expressão de proteínas ("Western Blotting")}

As amostras de músculo coletadas foram congeladas e mantidas em freezer $-80^{\circ} \mathrm{C}$ até serem homogeneizadas e utilizadas. O preparo para a análise de expressão de proteínas consistiu na homogeinização das amostras tampão de extração contendo (Trizma base $100 \mathrm{mM}$, SDS $10 \%$, para-hidroximercuriobenzoato $(\mathrm{p}-\mathrm{OHHgBz}) 1$ $\mathrm{mM}$; fenilmetilsulfonilfluoreto (PMSF) $1 \mathrm{mM}$; pepstatina A $1 \mathrm{mM}$; orto-fenantrolina $30 \mathrm{mM}$ e ácido etilendinitrolotetracético (EDTA) $(10 \mathrm{mM})$ e colocadas em banho a $100^{\circ} \mathrm{C}$ por $10 \mathrm{~min}$. O homogeneizado do músculo foi mantido no gelo, rapidamente centrifugado (3.000 rpm x $10 \mathrm{~min}$ ) e o sobrenadante armazenado a $-20{ }^{\circ} \mathrm{C}$. Parte do sobrenadante foi utilizado para quantificar a concentração total de proteínas (BRADFORD, 1976). Cada amostra foi diluída em tampão Laemmli (LAEMMLI, 1970) na proporção de 1:4 contendo DDT (Ditiotreitol) 100 $\mathrm{mM}$ e aquecidos em água fervente por $5 \mathrm{~min}$. Cada amostra contendo o Laemmli mais o precipitado foi submetida a uma rotação ("spin") de $4000 \mathrm{rpm}$ por 30 segundos e o sobrenadante foi submetido à eletroforese em gel de poliacrilamida (SDS- sodium dodecyl sulfate, PAGE 10\%) no aparelho para minigel (Mini-Protean, Bio- Rad, Hercules, CA,
EUA). Em cada gel foi aplicado como padrão um marcador de peso molecular com valores estabelecidos em: miosina (205-195 kDa), $\beta$ galactosidase $(116 \mathrm{kDa})$, albumina bovina $(80 \mathrm{kDa})$ e ovalbumina $(49,5 \mathrm{kDa})$.

\section{"Immunoblotting"}

A transferência das proteínas separadas no gel foi feita eletricamente para uma membrana de nitrocelulose utilizando-se o sistema "semi-dry" (TransBlot Semi-Dry Transfer Cell - Bio-Rad, Hercules, CA, EUA) e durou por volta de 40 minutos sob 20 volts, conforme descrito por Polvino, Saravis, Sampson e Cook (1983). No tampão usado para realizar a transferência foi acrescentado SDS 0,1\% para melhorar a eluição das proteínas de alto peso molecular. A ligação inespecífica de proteínas na membrana de nitrocelulose foi inibida pela incubação destas com $10 \mathrm{ml}$ de solução bloqueadora (leite desnatado Molico $5 \%$, Tris $10 \mathrm{mM}$, $\mathrm{NaCl} 150 \mathrm{mM}$ e Tween $200,02 \%)$ por $2 \mathrm{~h}$ na temperatura ambiente. As membranas com a amostra foram incubadas com o anticorpo primário para VEGF (SC152 da Santa Cruz Biotechnology; Santa Cruz, CA, EUA, diluição de 1:500) diluído em solução bloqueadora (leite desnatado 3\%, Tris $10 \mathrm{mM}, \mathrm{NaCl}$ $150 \mathrm{mM}$ e Tween $200,02 \%$ ) e incubadas a $4{ }^{\circ} \mathrm{C}$ "overnight". No dia seguinte, foram incubadas por 23 horas com o anticorpo secundário "anti-rabbit" (Zymed laboratories, South San Francisco, CA, EUA, 1:5000) e posteriormente foram lavadas três vezes por 10 minutos com solução basal. As bandas de proteínas específicas existentes nas membranas incubadas foram visualizadas através do uso do "Kit" para detecção por quimiluminescência (ECL-General Eletric Healthcare, Little Chalfont, Buckinghamshire, England). A seguir os filmes de raio-x foram expostos às membranas por aproximadamente 15 minutos. Para se medir a intensidade das bandas nas auto-radiografias, as figuras escaneadas foram analisadas utilizando o programa de análise de densitometria óptica Scion Image, fornecido gratuitamente pela NIH (USA) via internet (http:// w w w . s c i o n c o r p.com / p a g e s / scion_image_windows.htm).

\section{Estatística}

Os dados estão expressos na forma de média \pm erro padrão da média. Foi utilizada análise de variância de dois fatores (ANOVA) para análises bioquímicas, moleculares, morfológicas e morfométricas. 
Para dados de fluxo sanguíneo e débito cardíaco foi utilizada ANOVA de dois fatores para medidas repetidas para as diferenças entre grupos.

\section{Resultados}

\section{Peso corporal dos animais}

A TABELA 1 mostra os dados de peso corporal dos animais antes e após a realização do protocolo experimental.
Após os tratamentos estatísticos foi realizado o teste Post-hoc de Duncan para significâncias estatísticas, tendo sido adotado grau de significância para $\mathrm{p}<0,05$.
$\mathrm{SC}=$ Sedentário Controle; $\mathrm{SA}=$ Sedentário Anabolizado;

TC = Treinado Controle; $\mathrm{TA}=$ Treinado Anabolizado.

* = diferença significante em relação aos grupos sedentários;

** = diferença significante em relação ao grupo $\mathrm{SC}$ e TC;

$\dagger=$ diferença significante em relação ao grupo $S A$; $p<0,05$.

$\mathrm{SC}=$ Sedentário Controle; $\mathrm{SA}=\mathrm{S}$ e de n t á ri o Anabolizado;

$\mathrm{TC}=$ Treinado Controle

$\mathrm{T} A=\mathrm{Tr}$ e i $\mathrm{n}$ a d o Anabolizado.

* $\quad=$ diferença significante em relação aos grupos sedentários ** = diferença significante em relação ao grupo SC e TC;

$\dagger=$ diferença significante em relação ao grupo SA.

$p<0,05$.
TABELA 1 - Efeito do protocolo de treinamento, da administração do EAA e da associação de ambos sobre o peso corporal inicial e final (PC inicial e PC final, frequência cardíaca - FC e pressão arterial média - PAM).

TABELA 1 - Efeito do protocolo de treinamento, da administração do EAA e da associação de ambos sobre o peso
corporal inicial e final (PC inicial e PC final, frequência cardíaca - FC e pressão arterial média - PAM)
\begin{tabular}{ccccc}
\hline \\
\hline SC & PC inicial $(\mathbf{g})$ & PC final $(\mathrm{g})$ & FC (bpm) & PAM (mmHg) \\
SA & $215 \pm 12$ & $440 \pm 8,2$ & $328 \pm 7,3$ & $107 \pm 2,1$ \\
TC & $218 \pm 4,5$ & $399 \pm 3,1^{*}$ & $308 \pm 18,1$ & $106 \pm 1,4$ \\
TA & $195 \pm 1,7$ & $394 \pm 1,1^{*}$ & $285 \pm 6,7^{* *}$ & $103 \pm 5,1$ \\
\hline
\end{tabular}

\section{Nível plasmático de testosterona}

Os resultados de nível de testosterona plasmático estão mostrados na FIGURA 2. Conforme esperado, a administração crônica de EAA causou um significante aumento na concentração de
No início do protocolo experimental não haviam diferenças no peso corporal entre os grupos, porém ao final do treinamento os grupos SA, TC e TA apresentaram peso corporal estatisticamente diminuído em relação aos animais SC, sem diferenças entre si. testosterona plasmática $(\mathrm{ng} / \mathrm{dl}, \mathrm{SA}-85,3 \pm 19,3$; TA $-102,6 \pm 18,3$ vs. SC $-13,4 \pm 6,5$; TC $-20,5 \pm$ $9,9)$. As concentrações plasmáticas de testosterona foram superiores nos grupos que receberam tratamento com a droga (EAA) a dizer SA e TA quando comparados aos grupos SC e TC.

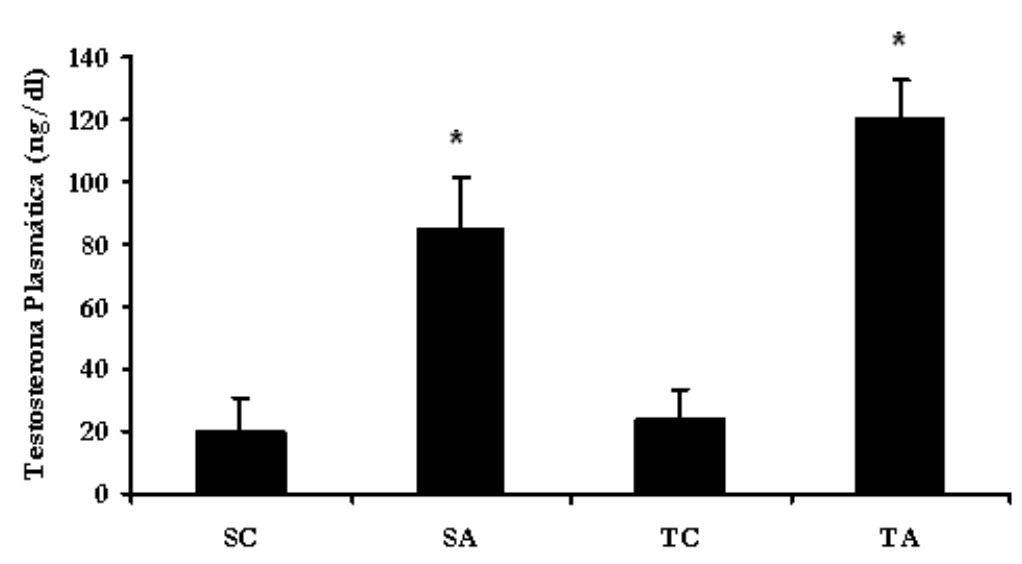

FIGURA 2 - Nível plasmático de testosterona após protocolo experimental. 


\section{Medidas hemodinâmicas}

Frequência cardíaca de repouso

Os dados de frequência cardíaca (FC) ao final do protocolo de treinamento estão demonstrados naTABELA 1. Pode-se observar que o treinamento físico promoveu redução da FC de repouso no grupo TC e TA.

\section{Pressão arterial média}

A pressão arterial média (PAM) não sofreu alteração significante entre os grupos, conforme indicado na TABELA 1.

\section{Débito cardíaco}

A FIGURA 3 mostra o débito cardíaco (DC) dos grupos experimentais na situação basal (DC basal) e após a administração de acetilcolina (DC Ach). O DC basal do grupo TA $(98 \pm 18 \mathrm{ml} / \mathrm{min})$ apresentou redução significativa comparado ao grupo TC $(150 \pm 36 \mathrm{ml} / \mathrm{min})$ e comparado aos grupos SA $(161 \pm 13 \mathrm{ml} / \mathrm{min})$ sem diferenças em relação ao grupo SC $(146 \pm 15 \mathrm{ml} / \mathrm{min})$. O valor de DC Ach do grupo TA permaneceu reduzido (85 $\pm 10 \mathrm{ml} / \mathrm{min}$ ), com redução significativa em relação ao grupo TC $(151 \pm 26 \mathrm{ml} / \mathrm{min}), S C(147 \pm 18$ $\mathrm{ml} / \mathrm{min})$ e $S A(145 \pm 20 \mathrm{ml} / \mathrm{min})$.

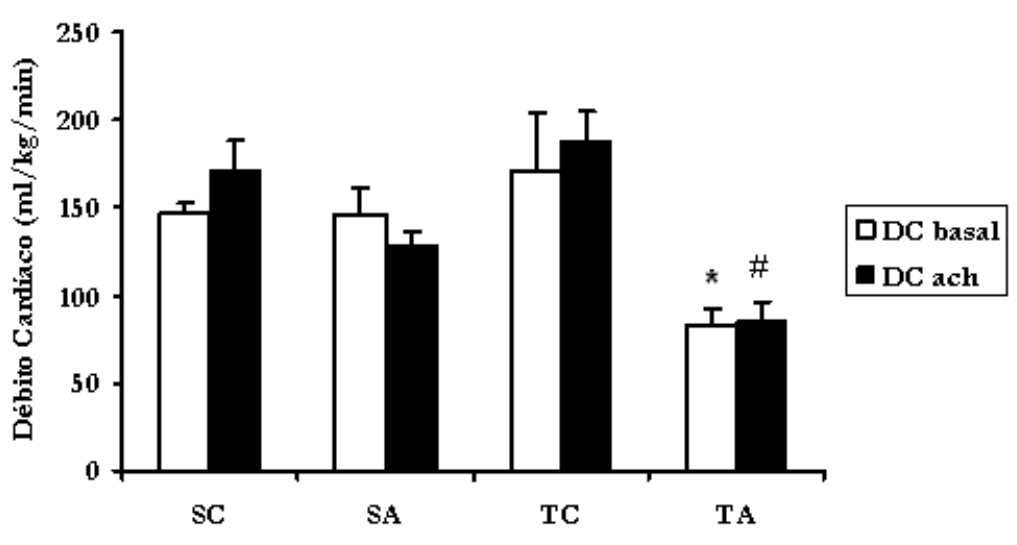

$\mathrm{SC}=$ Sedentário Controle; $\mathrm{S} A=\mathrm{S}$ e de n t á r i o Anabolizado; TC = Treinado Controle TA=Treinado Anabolizado. * = Diferença significativa em relação ao grupo TA basal;

\# = Diferença significativa em relação ao grupo TA sob administração de acetilcolina $(p<$ $0,05)$.

FIGURA 3 - Débito cardíaco (DC) basal e após administração de acetilcolina (ml/min).

\section{Fluxo sanguíneo}

A FIGURA 4 A mostra os resultados de perfusão regional $(\mathrm{Q})$ do músculo sóleo na condição basal $(\mathrm{Q}$ basal) e sob administração de acetilcolina (Q Ach). Não houve diferença significativa entre os grupos para os valores de $\mathrm{Q}$ basal. Após a administração de acetilcolina houve diminuição significativa dos grupos TA $(0,82 \pm 0,20 \mathrm{ml} / \mathrm{min})$ e SC $(1,20 \pm 0,15 \mathrm{ml} / \mathrm{min})$ em relação ao grupo TC $(2,60 \pm 0,39 \mathrm{ml} / \mathrm{min})$. Os animais TC tiveram $\mathrm{Q}$ Ach significativamente aumentada em relação à sua $Q$ basal $(0,79 \pm 0,24 \mathrm{ml} /$ $\mathrm{min}$ ), o que não ocorreu com os outros grupos.

Este aumento não foi observado nos grupos sedentários, e o grupo TA apresentou uma diminuição significativa de Q Ach em relação ao grupo TC, não apresentando diferenças quando comparado a ambos os grupos sedentários (SC e SA). 
$\mathrm{SC}=$ Sedentário Controle; $S A=S$ e de n t á rio Anabolizado; TC = Treinado Controle TA=TreinadoAnabolizado * = diferença significativa dos animais TC sob administração de acetilcolina em relação aos grupos TA e SC; \# = diferença significativa do grupo TC em relação à sua condição basal. $(p<0,05)$

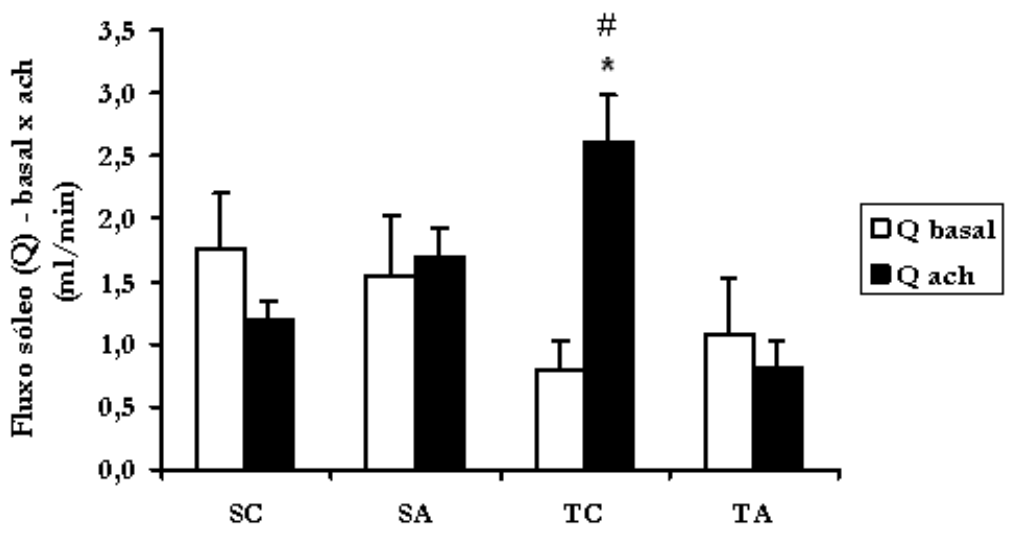

FIGURA 4 - Fluxo sanguíneo basal (Q basal) e sob administração de acetilcolina (Q Ach) para o músculo sóleo.

Morfologia muscular esquelética Hipertrofia

Os resultados da avaliação de hipertrofia da musculatura esquelética obtidos pelo peso do músculoesquelético normalizado pelo peso corporal do animal (sóleo: $\mathrm{SC}=0,49 \pm 0,02, \mathrm{SA}=0,48 \pm 0,01, \mathrm{TC}=0,44$ $\pm 0,03, \mathrm{TA}=0,48 \pm 0,01 \mathrm{mg} / \mathrm{g})$ mostram que nem o TFN, nem o uso de EAA foram eficazes em causar hipertrofia no músculo estudado.

Morfometria muscular esquelética Número de capilares por fibra

Os resultados da análise histológica para sóleo estão apresentados na FIGURA 5. Foram encontrados aumentos significativos na razão capilar/fibra (rc/f) do grupo TC $(2,29 \pm 0,08)$ comparado aos grupos TA $(1,82 \pm 0,09)$, SC $(1,74 \pm 0,12)$ e SA $(1,58 \pm 0,07)$, ou seja, o número de capilares por fibra muscular esquelética encontra-se aumentado no grupo TC em relação aos outros grupos para o músculo estudado.

\section{Expressão de VEGF}

("Vascular-Endotelial Growth Factor")

A FIGURA 6 mostra os resultados de expressão de VEGF para sóleo, corrigidos pelos respectivos resultados de expressão da proteína normalizadora, a Gliceraldeído 3-fosfato desidrogenase (GAPDH Glyceraldehyde 3-phosphate dehydrogenase). Não houve diferença estatística entre os grupos para a proteína normalizadora GAPDH. Para a expressão de VEGF, expressa como Razão VEGF/GAPDH houve aumento significativo aumentou no grupo TC $(1,75$ $\pm 0,25$ ) comparado aos outros grupos, ou seja, a expressão do VEGF se encontra 31\% aumentada neste grupo em relação aos grupos TA $(1,02 \pm 0,08), 58 \%$ em relação ao grupo SC $(0,73 \pm 0,09)$ e $39 \%$ em relação ao grupo $S A(1,07 \pm 0,13)$.
$\mathrm{SC}=$ Sedentário Controle; $S A=S$ ed en tá ri o Anabolizado;

$\mathrm{TC}=$ Treinado Controle

$\mathrm{TA}=$ Treinado Anabolizado.

* = Aumentos significativos para o grupo TC comparado aos grupos TA, SA e SC.

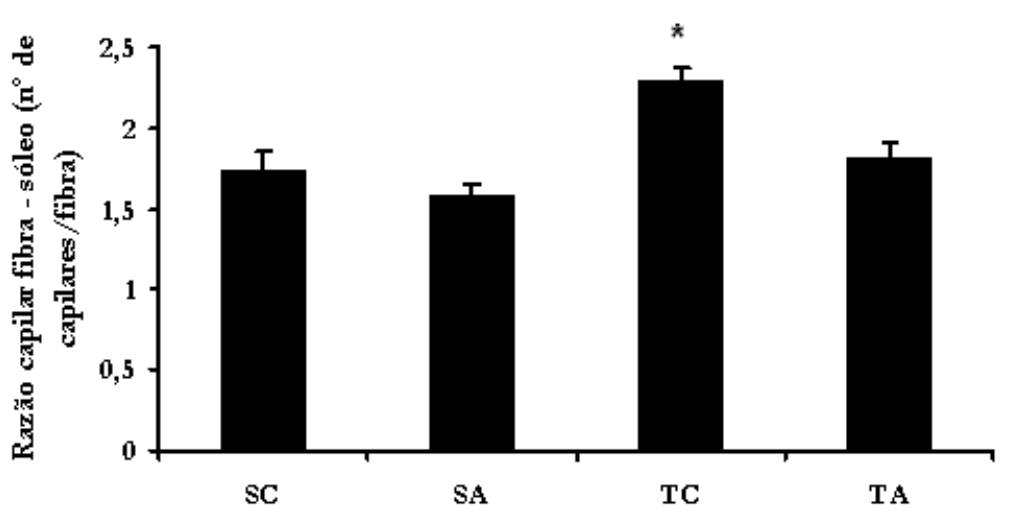

FIGURA 5A - Razão capilar/fibra para o músculo sóleo. 
Esteróide anabolizante

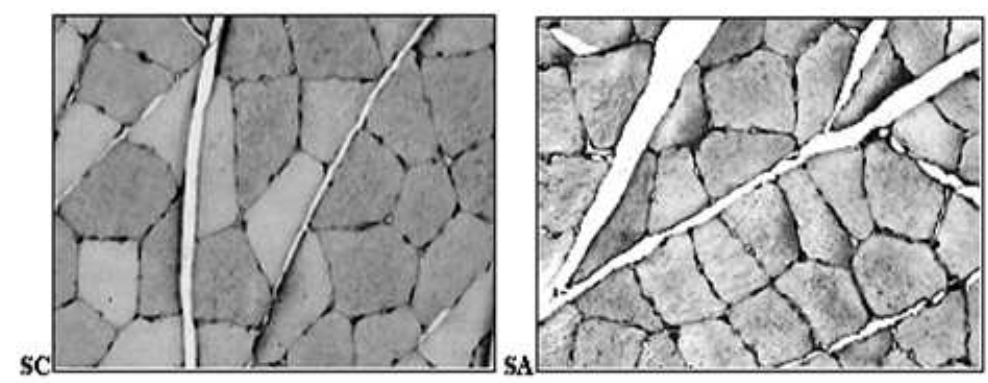

$\mathrm{SC}=$ Sedentário Controle; $\mathrm{SA}=\mathrm{S}$ e de n tário Anabolizado;

TC = Treinado Controle

TA=TreinadoAnabolizado.

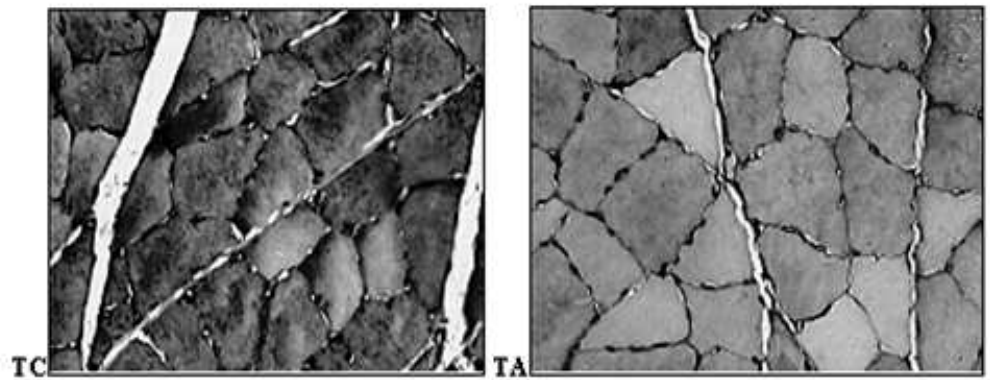

FIGURA 5B - Fotos ilustrativas dos campos observados através de microscopia ótica do músculo sóleo através de lentes com aumento de 40x para os quatro grupos.
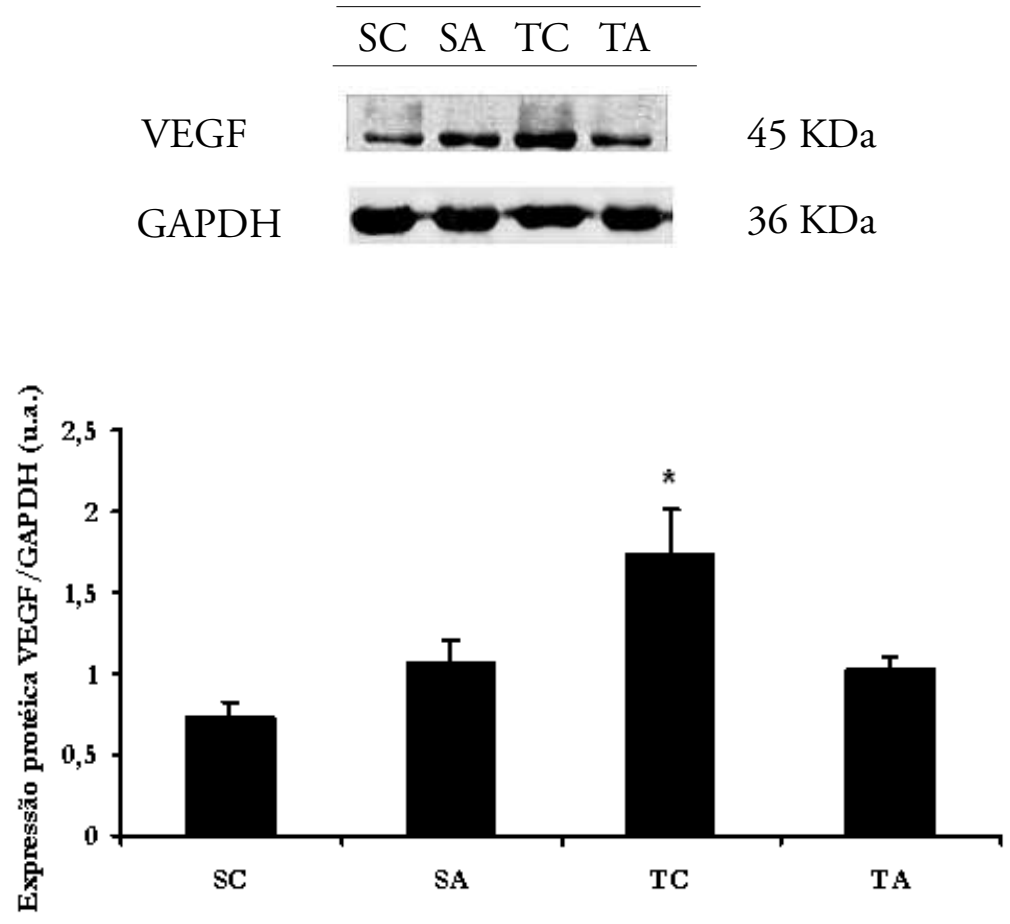

$\mathrm{SC}=$ Sedentário Controle; $S A=S$ e de n t á ri o Anabolizado;

TC = Treinado Controle $\mathrm{TA}=$ Treinado Anabolizado. Resultados expressos como média \pm erro padrão. $(p<0,05)$.

* $=$ Aumentos significativos do grupo TC comparado aos grupos $\mathrm{TA}$, $\mathrm{SC}$ e SA. $p<0,05$.

FIGURA 6 - Expressão proteíca de VEGF e GAPDH para sóleo (Western Blot): razão VEGF/GAPDH para o músculo sóleo. 


\section{Discussão}

Os principais resultados encontrados nesse estudo demonstram que o uso de doses suprafisiológicas de EAA associado ao treinamento de natação provoca diminuição da resposta vasodilatadora endotélio dependente (Q Ach), no sóleo, devido ao prejuízo de fluxo sob administração de Ach. Este prejuízo se reflete na expressão de VEGF, gerando diminuição no número de capilares por fibra muscular esquelética no músculo estudado.

O presente estudo mostrou que a administração de EAA causa um aumento na concentração de testosterona plasmática nos animais TA e SA. Estes resultados são consistentes com efeitos descritos previamente com a concentração de testosterona plasmática mais alta em ratos submetidos a tratamento crônico com EAA (TRIfUNOVIC, NorTON, Duffield, Avraam \& Woodiwiss, 1995).

Houve redução na progressão do PC no decorrer do protocolo. Os grupos TC, SA e TA apresentaram menor PC final em relação aos animais do grupo SC. Esses resultados refletem um maior metabolismo de lipídeos alcançado pelo treinamento aeróbio e pela administração do EAA. Os resultados apresentados neste estudo estão em acordo com os resultados obtidos por RochA et al. (2007), que demonstraram um menor peso corporal ao final do protocolo experimental, nos animais do grupo SA, TC e TA, quando comparados ao SC. Esta diferença de peso foi relacionada à redução da gordura intraperitonial destes grupos.

Resultados similares foram descritos em estudos onde o excesso de andrógenos causou um aumento no metabolismo de lipídeos no tecido adiposo o que resulta em menor ganho de peso (BEUTEL, BERGAMASCHI \& CAMPOS, 2005; SCHROEDERZHENG, Ong, Martinez, Flores, Stewart, Azen \& Sattler, 2004; Yu-Yahiro, Michael, Nasralah \& SHOfield, 1989). Esses resultados ainda são controversos na literatura, pois existem estudos que não demonstraram diminuições nem acréscimos no peso corporal (MASONIS \& MCCARTHY, 1995), o que parece ser reflexo do tipo de droga utilizada, dosagem e protocolo experimental empregado.

Os resultados de peso do músculo corrigido pelo peso corporal sugerem que não ocorreu hipertrofia no músculo estudado. Estes resultados são condizentes com os efeitos do treinamento aeróbio, em intensidades baixas, na área de secção transversa do músculo, que tem um potencial hipertrófico diminuído em relação a treinamentos mais intensos e a músculos com predominância de fibras brancas (Maughan, Gleeson \& Greenhaff, 2000).
A bradicardia de repouso dos animais TA e TC confirma a efetividade do treinamento físico aeróbio neste estudo, como já relatado previamente por outros estudos realizados em nosso laboratório (REDONDO, 2007; Rocha et al., 2007). Esta adaptação ao exercício crônico foi mantida com o tratamento com EAA.

O DC do grupo TA se encontrou diminuído em relação aos outros grupos, em condições basais (DC basal) e sob administração de acetilcolina (DC Ach). Dados já publicados relatam que doses suprafarmacológicas de EAA geram diminuição da resposta inotrópica do coração devido à presença de hipertrofia patológica e aumento de fibrose por deposição de colágeno tipo I e III (RocHA et al., 2007). É possível que a associação dos efeitos deletérios citados anteriormente, oriundos da administração da droga em conjunto à bradicardia de repouso, observada também no grupo TA, resulte em uma diminuição significativa de DC, no grupo TA, tanto por diminuir o volume sistólico, em decorrência de prejuízo inotrópico, quanto por redução da FC, em decorrência do treinamento. Este efeito que é normalmente considerado como uma adaptação benéfica sem o uso da droga, devido a um aumento compensado no volume sistólico (VS), parece nocivo para o usuário de EAA que o associa ao treinamento aeróbio, no qual uma diminuição da $\mathrm{FC}$ não é compensada pelo aumento no VS.

Em condição basal ( $Q$ basal) não foi observada alteração na perfusão regional para o músculo sóleo entre os grupos. No entanto, é importante ressaltar que, devido à diminuição do DC observada no grupo TA esta condição pode refletir numa diminuição perfusional de outros sistemas orgânicos, que provavelmente operam de forma deficitária de oxigênio e substratos em função da diminuição de fluxo oriunda da diminuição do DC.

Ao avaliar a vasodilatação endotélio dependente com a infusão por Ach em músculos sóleo, o grupo TC obteve aumento de fluxo sanguíneo em relação aos grupos SC, SAeTA. Adicionalmente, neste músculo, que apresenta predomínio do metabolismo oxidativo o grupo TC apresentou Q Ach aumentado em relação sua condição basal (Q basal) o que não foi observado nos outros grupos. Ambos os resultados mostram uma capacidade vasodilatadora endotélio dependente aumentada em função do efeito do treinamento sobre a vasculatura muscular. A função endotelial é essencial para a manutenção do fluxo sanguíneo, e para o controle vasomotor de vasos de condutância e resistência e o 
exercício crônico aumenta a vasodilatação endotelial dependente de $\mathrm{NO}$ em ambos os vasos (GREEN, Maiorana, O’Driscoll \& TAYLOR, 2004).

Por outro lado, é passível de observação que os grupos SC e SA não apresentaram diferenças no fluxo sob ação da Ach (Q Ach) em relação à sua condição basal (Q basal). É possível que isto tenha ocorrido em função de uma menor sensibilidade à Ach destes grupos, não por apresentar prejuízo na sua capacidade vasodilatadora endotélio dependente, mas provavelmente porque, devido a não ser submetido ao TFN, perifericamente não apresenta uma capacidade vasodilatadora endotélio dependente desenvolvida o suficiente para responder à quantidade de Ach infundida, sendo que no sóleo, para estes grupos, não apresentam características adaptativas ao treinamento, como os do grupo TC. Contrariamente ao estudo de REDONDO (2007), que observou aumento de $\mathrm{Q}$ Ach dos animais $\mathrm{SC}$ em relação à $\mathrm{Q}$ basal no coração, este estudo mostra que os animais sedentários podem não apresentar resposta vasodilatadora pronunciada em relação a sua condição basal, porque seu fenótipo não está adaptado ao treinamento. Ainda, como o experimento das esferas é realizado com o animal em repouso, o músculo esquelético não constitui em um sistema prioritário como o coração nesta situação. Estes resultados são compatíveis com outro estudo que mostra que tanto a sensibilidade quanto a resposta máxima à Ach de roedores sedentários está diminuída, e que ambas são aumentadas com o treinamento em esteira (McAllister, Newcomer \& Laughin, 2008).

No grupo TA após a infusão de Ach foram observados os maiores prejuízos sobre vasculatura músculo-esquelética, evidenciando as consequências do abuso do EAA associado ao treinamento aeróbio. A resposta vasodilatadora dependente de endotélio deste grupo foi inibida no músculo sóleo, o que sugere que a associação de doses abusivas de EAA com o treinamento aeróbio apresenta resposta diminuída à Ach, com capacidade vasodilatadora dependente de endotélio prejudicada em relação ao grupo que treinou e não recebeu o EAA. A capacidade de vasodilatação endotélio dependente é um aspecto importante durante o exercício, permitindo um aporte adequado de sangue e substratos para os músculos ativos e para manter a saúde vascular (GREEN et al., 2004).

$\mathrm{O}$ estímulo físico do aumento de fluxo como ocorre, por exemplo, durante o exercício físico é capaz de disparar a biossíntese de fatores de crescimento angiogênicos protetores contra doenças vasculares (Prior, Yang \& Terjung, 2004). Com a resposta de fluxo sob ação de Ach prejudicada no grupo TA, provavelmente durante o exercício existe diminuição da perfusão através do controle dos vasos de resistência (arteríolas), com prejuízo da reatividade vascular e também diminuição do estresse de cisalhamento no lúmen dos vasos, o que prejudica a angiogênese induzida pelo exercício.

Os resultados deste estudo são compatíveis com o de CunHA etal. (2005), que mostra que a menor sensibilidade de anéis de aorta de ratos à substância vasoconstritora fenilefrina obtida pelo treinamento anaeróbio foi revertida pelo grupo treinado e tratado com nandrolona, o que sugere que o tratamento com o EAA gera prejuízo em algum mecanismo importante envolvido nos processos de vasodilatação dependente de endotélio, como a biossíntese de óxido nítrico (NO), por exemplo.

Adicionalmente, o TFN é um estímulo que pode gerar o alargamento de um vaso arterial (arteriogênese) em resposta ao fluxo sanguíneo aumentado. A elevação do estresse de cisalhamento na superfície endotelial gera não apenas uma distensão na parede do vaso devido a uma pressão intraluminal aumentada, mas um aumento em seu diâmetro e dimensões da parede, decorrente do remodelamento de células endoteliais, musculares lisas e de fibroblastos. Este estímulo tem relação com o aumento do estresse de cisalhamento no lúmen arterial, causado pelo aumento do fluxo circulante, sendo que o vaso se alarga para normalizálo respondendo com um espessamento da parede. Ao contrário, se o fluxo é reduzido na resposta ao exercício o diâmetro da artéria se reduz, em resposta a um menor estresse de cisalhamento e o aporte de sangue é reduzido para o tecido (Prior, YANG \& TerJung, 2004)

Adicionalmente, os resultados mostram que o EAA associado ao TFN, atenuou o aumento no número de capilares por fibra muscular esquelética, sendo a angiogênese como efeito do treinamento inibida no grupo TA, que apresentam os valores de rc/f similar aos valores dos grupos sedentários (SC e SA). O prejuízo apresentado pelo músculo sóleo do grupo TA no Q Ach, ao invés de ser compensado por um aumento no número de capilares por fibra muscular, foi agravado por uma proliferação inibida no grupo TA, o que reforça a hipótese de que esta associação é capaz de gerar anormalidades na resposta vascular ao exercício no músculo estudado (De Angelis, OGAWA, Sanches, Rigatto, Krieger \& Irigoyen, 2006; Soares $\&$ Duarte, 1991; Tagarakis et al., 2000).

Além da diminuição do fluxo regional derivada de um provável prejuízo na vasodilatação dependente de endotélio, e contrariamente ao grupo de animais TC, foi observada rarefação de capilares nos 
músculos estudados no grupo TA. Existem estudos que mostram resultados similares no leito capilar cardíaco e muscular esquelético de ratos submetidos a protocolos que associam treinamento em esteira e EAA (Dimauro, Balnave \& Shorey, 1992; Hudlicka, Dodd, Renkin \& Grey, 1982; Soares $\&$ DuARTE, 1991; TAgarakis et al., 2000).

A análise da expressão protéica de VEGF confirma os resultados de angiogênese no grupo TC, além de explicar a rarefação no grupo TA comparado ao grupo TC, apresentando adicionalmente valores similares aos grupos SC e SA. Há aumentos de expressão de VEGF e de valores de $\mathrm{r}$ c/f no grupo TC sem alteração para TA, SC e SA.

É preciso considerar que o grupo TA apesar de ser submetido ao treinamento de natação apresenta valores iguais ( $\mathrm{r}$ c/f e VEGF) que os grupos SC e SA. Estes resultados sugerem que apesar de estar sendo submetido ao estresse do exercício físico, a capacidade adaptativa microvascular, que é benéfica à saúde e decorrente do exercício crônico está prejudicada. Os grupos SC e SA não apresentam esta alteração, o que é justificado pelo fato dos mesmos não serem submetidos ao TFN. O grupo TA sofre prejuízo funcional e estrutural sem as adaptações benéficas causadas pelo TFN por si só, importantes para que os efeitos do estresse sobre a musculatura possam ser compensados e sejam benéficos à saúde, já que além de apresentarem capacidade vasodilatadora endotélio dependente diminuída apresentam também uma menor expressão de VEGF, e uma atenuação não esperada para um animal treinado na quantidade de capilares funcionais por fibras musculares esqueléticas.

VEGF é considerado o principal fator de crescimento envolvido na angiogênese e sua expressão é iniciada pela hipóxia e fortemente estimulada pelo estresse de cisalhamento decorrente do aumento do fluxo sanguíneo nas sessões de exercício durante o treinamento aeróbio (Prior, Yang \& Terjung, 2004). No grupo TA sua expressão se encontra diminuída em relação ao grupo TC sem diferenças em relação aos grupos sedentários.

A atenuação dos valores de $\mathrm{r}$ c/f e da expressão de VEGF foi consistente no músculo estudado, indicando que o grupo TA, além de apresentar DC e Q Ach diminuído, também apresenta uma menor quantidade de capilares por fibra muscular esquelética, que parece ser uma consequência relacionada à diminuição do fluxo, aliada ao advento de diminuição na expressão de VEGF e suas vias de sinalização.

Diversos estudos confirmam o papel de VEGF na angiogênese induzida pelo exercício mostrando aumentos nos níveis de mRNA e de expressão protéica de VEGF em ratos e relacionando estes aumentos a uma quantidade aumentada de capilares funcionais (Amaral, Linderman, Morse \& Greene, 2001; Amaral, Papanek \& Greene, 2001; Kraus et al.; 2004; Milkiewics, Hudlicka, Brown \& Silgran 2005), resultados que coincidem nesse estudo com os aumentos de expressão de VEGF e de r c/f do músculo estudado no grupo TC. O reconhecimento de que há uma responsividade melhorada para o remodelamento microvascular de indivíduos treinados comparados a sedentários pode contribuir para a associação geral entre um estilo de vida ativo e menor incidência de doenças vasculares.

Contrariamente, os resultados do grupo TA sugerem que associar EAA ao TFN gera atenuação da angiogênese ocasionada pelo treinamento de resistência aeróbia por si só, o que leva a crer que esta associação é deletéria para o endotélio e para os capilares no músculo esquelético, o que predispóe o praticante de esportes e atividades físicas a problemas vasculares. O estudos de D'Ascenzo, Millinagi, Di Masimo, Saccani-Jotti, Botrè, Carta, Tozzi-Ciancarelli, Pavan e Dolo (2006) e Ray, Herring, Markel, Crisostomo, Wang, Weil, Lahm e Meldrum (2008), ambos empregando cultura de células, mostram "in vitro" os efeitos deletérios da testosterona e seus derivados em doses suprafisológicas em células endoteliais da veia umbilical e na produção de VEGF em células tronco mesenquimais de ratos. Estes reforçam a hipótese de que os EAA são capazes de alterar a homeostase das células endoteliais e que podem diminuir a produção de fatores de crescimento que protegem contra doenças vasculares. Esses estudos mostram que a exposição a doses suprafisiológicas de EAA altera o crescimento das células endoteliais com um forte efeito antiproliferativo, induzindo apoptose e modificando as concentraçôes intracelulares de cálcio, um marcador para ativação precoce de células endoteliais, que provoca a produção e lançamento de citocinas e fatores predisponentes a dano vascular (WatANABE, TANAKA, Wen \& Yoshida, 1996). Os resultados do presente estudo sugerem que é possível a ocorrência de efeitos similares "in vivo" no músculo sóleo, ocasionando prejuízo na reatividade vascular que culminaram na diminuição no número de capilares funcionais e perda do efeito de treinamento para o grupo que associou altas doses de EAA e o treinamento aeróbio.

É interessante elucidar no futuro quais mecanismos estão envolvidos nessa atenuação gerada pela associação 
de EAA e treinamento físico no músculo esquelético com diferentes predominâncias de fibras, além da participação do VEGF, já que o envolvimento de diversos outros fatores de crescimento, vias de sinalização e suas interações coordenadas na angiogênese induzida pelo exercício atualmente são reconhecidos na literatura (CONWAY, COLlen \& Carmeliet, 2001; Prior, Yang \& Terjung, 2004).

Finalmente, este estudo mostra evidência de que a associação entre EAA e TFN é deletéria para o endotélio e para a microvasculatura no músculo sóleo, o que tem relação com a diminuição na expressão de VEGF comparado ao resultado observado como efeito do treinamento físico por si só. Esta diminuição está relacionada à diminuição do fluxo sanguíneo muscular em resposta ao exercício, que provavelmente é uma consequência da diminuição do débito cardíaco em repouso e durante o exercício. Desta forma, esse estudo mostra que não é possível garantir manutenção da saúde vascular para usuários de EAA mesmo quando associados à atividade física.

\begin{abstract}
Anabolic steroid impairs the angiogenesis induced by swimming training in soleus muscle of normotensive rats

Androgenic anabolic steroids (EAA) are synthetic derivatives of testosterone, used in therapeutic dosages in medical practice and in high doses by physically active people that could be health damaging. To study the effects of EAA on the cardiovascular system, Wistar rats were randomized into Sedentary Control (SC), Sedentary Steroid (SA), Trained Control (TC) and Trained Steroid (TA) groups. We evaluated the effects of swimming training (60min/day, 5x/week during 10 week) and AAS (nandrolone decanoate - $5 \mathrm{mg} / \mathrm{kg} \mathrm{sc}, 2 x /$ week) on cardiac output, basal blood flow ( $Q b, D C$ basal) and after injection of a vasodilator to observe the endothelium dependent vasodilatation (acetylcholine - 0 Ach)( $Q$ Ach, DC Ach), capillary to fiber ratio ( $\mathrm{r} / \mathrm{f}$ ) and vascular-endothelial growth factor expression (VEGF) in soleus muscle (oxidative fibers). Serum testosterone increased in SA and TA. Exercise training significantly decreased resting heart rate. $\mathrm{Qb}$ was not different among groups, and QAch was higher in TC group, however in TA group this beneficial effect of swimming exercise training was lost by association with EAA. Rc/f and VEGF were higher only in TC group. These results suggest that swimming training associated with EAA inhibit angiogenesis and arteriogenesis observed as effects of aerobic training, and impairs the red skeletal muscle blood flow which predispose physically active AAS users to vascular diseases.
\end{abstract}

UNITERMS: Doping; Blood flow; Capillary; Skeletal muscle; Swimming training.

\title{
Referências
}

AMARAL, S.L.; PAPANEK, P.E.; GREENE A.S. Angiotensin II and VEGF are involved in angiogenesis induced by short-term exercise training. American Journal of Physiology: Heart and Circulatory Physiology, Bethesda, v.281, p.H1163-9, 2001.

AMARAL, S.L.; LINDERMAN, R.J.; MORSE, M.M.; GREENE, A.S. Angiogenesis induced by electrical stimulation is mediated by angiotensin II and VEGF. Microcirculation, New York, v.8, p.57-67, 2001

ANDERSEN, R.E.; BARTLETT, S.J.; MORGAN, G.D.; BROWNELL, K.D. Weight loss, psychological and nutritional patterns in competitive male body builders. International Journal of Eating Disorders, New York, v.18, n.1, p.49-57, 1995. BEUTEL, A.; BERGAMASCHI, C.T., CAMPOS, R.R. Effects of chronic anabolic steroid treatment on tonic and reflex cardiovascular control in male rats. The Journal of Steroids Biochemistry and Molecular Biology, Oxford, v.93, p.43-8, 2005. BORNES, S.; BOULARD, M.; HIEBLOT, C.; ZANIBELLATO, C.; IACOVONI, J.S.; PRATS, H.; TOURIOL, C. Control of the vascular endothelial growth factor internal ribosome entry site (IRES) activity and translation initiation by alternatively spliced coding sequences. The Journal of Biological Chemistry, Baltimore, v.279, n.18, p.18717-26. 2004. 
BRADFORD, M.M. A rapid and sensitive method for the quantization of microgram quantities of protein utilizing the principle of protein-dye binding. Analytical Biochemistry, New York, v.72, p.248-54, 1976.

CELOTTI, F.; CESI, P.N. Anabolic steroids: a review of their effects on the muscle, of their possible mechanisms of action and of their use in athletics. The Journal of Steroids Biochemistry and Molecular Biology, Oxford, v.43, p. 46977, 1992.

COLÉGIO BRASILEIRO DE EXPERIMENTAÇÃO ANIMAL (COBEA). Princípios éticos na experimentação animal, 1991. Disponível em: <http://cobea.org.br/ética/htm\#3>. Acesso em: set. 2006.

CONWAY, E.M.; COLLEN, D.; CARMELIET, P. Molecular mechanisms of blood vessel growth. Cardiovascular Research, London, v.49, p.507-21, 2001.

COSTILL, D.L.; MAGLISCHO, E.W.; RICHARDSON, A.B. Handbook of sports medicine and science-swimming, an IOC medical commission publication, FINA. Oxford: Blacwell Science, 1992. cap.12, p.133-43.

CUNHA, T.S.; MOURA, M.J.C.S.; BERNARDES, C.F.; TANNO, A.P.; MARCONDES, F.K. Vascular sensivity to phenylephrine in rats submitted to anaerobic training and nandrolone treatment. Hypertension, Dallas, v.46, p.1010-5, 2005.

D’ASCENZO, S.; MILLIMAGI, D.; DI MASSIMO, C; SACCANI-JOTTI, G.; BOTRĖ, F.; CARTA, G.; TOZZICIANCARELLI, M.J.; PAVAN, A.; DOLO, V. Detrimental effects of anabolic steroids on human endothelial cells. Toxicology Letters, Amsterdam, v.169, n.2, p.129-36, 2006.

DE ANGELIS, K.; OGAWA, T; SANCHES, I.C.; RIGATTO, K.V.; KRIEGER, E.M; IRIGOYEN, M.C. Impairment on cardiac output and blood flow adjustments to exercise in L-NAME-induced hypertensive rats. Journal of Cardiovascular Pharmacology, New York, v.47, p.371-6, 2006.

DIMAURO, J.; BALNAVE, R.J.; SHOREY, C.D. Effects of steroids and high intensity exercise on rat skeletal muscle fibres and capillarization. A morphometric study. European Journal of Applied Physiology and Occupational Physiology, Berlin, v.64, p.204-12, 1992.

FULLER, M.G. Anabolic-androgenic steroids: use and abuse. Comprehensive Therapy, Harvard, v.19, p.69-72. 1993. GREEN, D.J.; MAIORANA, A.; O’DRISCOLL; TAYLOR, R. Effect of exercise training on endothelium-derived nitric oxide in humans. The Journal of Physiology, London, v.561, p.1-25, 2004.

HAKKINEN, J.P.; MILLER, M.W.; SMITH, A.H.; KNIGHT, D.R. Measurement of organ blood flow with colored microspheres in rat. Cardiovascular Research, London, v.29, p.74-9, 1995.

HEBERT, A.; HAUPT, M.D.; GEORGE, D.; ROVERE, M. Anabolic steroids: a review of the literature. The American Journal of Sports Medicine, Baltimore, v.12, p.469-84, 1984.

HUDLICKA, O.; DODD, L.; RENKIN, E.M.; GRAY, S.D. Early changes in fiber profile and capillaries in various muscles. Respiratory Physiology, Amsterdam, v.44, p.87-111, 1982.

INGJER, F; BRODAL, P. Capillary supply of skeletal muscle fibers in untrained and endurance-trained women. European Journal of Applied Physiology, Berlin, v.38, p.291-9, 1978.

JENSEN, L.; BANGSBO. J.; HELLSTEN, Y. Effect of high intensity training on capillarization and presence of angiogenic factors in human skeletal muscle. The Journal of Physiology, London, v.557(Pt 2), p.571-82, 2004.

KAM, P.C.A.; YARROW, M. Anabolic steroid abuse: physiological and anaesthetic considerations. Anaesthesia, London, v.60, p.685-92, 2005.

KINDERMANN, W. Cardiovascular side effects of anabolic-androgenic steroids. Herz, Munchen, v.31, p.566-73, 2006.

KUHN, C.M. Anabolic steroids. Recent Progress in Hormone Research, New York, v.57, p.411-34, 2002.

KRAUS, R.M.; STALLINGS, H.W.; YEAGER, R.C.; GAVIN, T.P. Circulating plasma VEGF response to exercise in sedentary and endurance-trained men. Journal of Applied Physiology, Washington, v.96, p.1445-50, 2004.

LAEMMLI, U.K. Cleavage of structural proteins during the assembly of the head of bacteriophage T4. Nature, London, v.227, n.259, p.680-5, 1970.

MASONIS A.E.; McCARTHY M.P. Direct effects of the androgenic/anabolic steroid stanozolol and 17 alphamethyltestosterone on benzodiazepine binding to the gamma-aminobutyric acid(a) receptor. Neuroscience Letters, Amsterdam, v.189, p.35-8, 1995.

McALLISTER, R.M.; NEWCOMER, S.C.; LAUGHLIN, M.H. Vascular nitric oxide: effects of exercise training in animals. Applied Physiology, Nutrition, and Metabolism, Otawa, v.33, n.1, p.173-8, 2008.

MANETTA, M.C.D.P.; SILVEIRA, D.X. Uso abusivo de anabolizantes androgênicos. Psiquiatria na Prática Médica, São Paulo, v.33, p.1-3, 2000.

MAUGHAN, R.; GLEESON, P.L.; GREENHAFF, P.L. Adaptação metabólica ao treinamento. In: Bioquímica do exercício e treinamento. Barueri: Manole, 2000. cap.8, p.179-211. 
MEDEIROS, A.; OLIVEIRA, E.M.; GIANOLLA, R.; CASARINI, D.E.; NEGRÃO, C.E.; BRUM, P.C. Swimming training increases cardiac vagal activity and induces cardiac hypertrophy in rats. Brazilian Journal of Medical and Biological Research, Ribeirāo Preto, v.37, p.1909-17, 2004.

MILKIEWICS, M.; HUDLICKA, O.; BROWN, M.D.; SILGRAN, H. Nitric Oxide, VEGF, and VEGFR-2: interactions in activity-induced angiogenesis in rat skeletal muscle. American Journal of Heart an Circulatory Physiology, Bethesda, v.289, p.H336-43, 2005.

POLVINO, W.J.; SARAVIS, C.A.; SAMPSON, C.E.; COOK, R.B. Improved protein analysis on nitrocellulose membrane. Electrophoresis, Weinheim, v.4, p.368, 1983.

POPE JUNIOR, H.G.; KATZ, D.L. Affective and psichotic syndromes associated with use of anabolic-androgenic steroids. American Journal of Psychiatry, Hanover, v.145, p.487-90. 1988.

PRIOR, B.M.; YANG, H.T.; TERJUNG, R.L. What makes vessels growth with exercise training? Journal of Applied Physiology, Washington, v.97, p.1119-28, 2004.

RAY, R.; HERRING, C.M.; MARKEL, T.A.; CRISOSTOMO P.R.; WANG, M.; WEIL, B.;LAHM, T.; MELDRUM, D.R. Deleterious effects of endogenous and exogenous testosterone on mesenchymal stem cell VEGF production. The American Journal of Physiology: Regulatory, Integrative and Comparative Physiology, Bethesda, v.294, p. R1498-1503, 2008.

REDONDO, F.R.R. Efeitos do uso de esteróides anabolizantes associados ao treinamento físico de natação sobre o fluxo sanguíneo para o miocárdio de ratos normotensos. 2007. 126 f. Dissertação (Mestrado em Bioquímica da Atividade Motora) - Escola de Educação Física e Esporte, Universidade de São Paulo, São Paulo, 2007.

ROCHA, F.L.; CARMO, E.C.;ROQUE,F.R.; HASHIMOTO, N. Y.; ROSSONI, L.V.; FRIMM, C.; ANÉAS, I.; NEGRÃO, C.E.; KRIEGER, J.E.; OLIVEIRA, E.M. Anabolic steroids induce cardiac renin-angiotensin system and impair the beneficial effects of aerobic training in rats. American Journal of Physiology: Heart and Circulatory Physiology, Bethesda, v.293, p.H3575-83, 2007.

SALTIN, B.; ROWELL, L.B. Functional adaptations to physical activity and inactivity. Federation Proceedings, Washington, v.39, p.1506-13, 1980.

SCHROEDER, E.T.; ZHENG, L.; ONG, M.D.; MARTINEZ, C.; FLORES, C.; STEWART, Y.; AZEN, C.; SATTLER, F.R. Effects of androgen therapy on adipose tissue and metabolism in older men. The Journal of Clinical Endocrinology and Metabolism, Springfield, v.89, p.4863-72, 2004.

SOARES, J.M.; DUARTE, J.A. Effects of training and an anabolic steroid on murine red skeletal muscle. A stereological analysis. Acta Anatomica, Basel, v.142, p.183-7. 1991.

TAGARAKIS, C.V.; BLOCH, W.; HARTMANN, G.; HOLLMANN, W.; ADDICKS, K. Anabolic steroids impair the exercise-induced growth of the cardiac capillary bed. International Journal of Sports Medicine, Stuttgart, v.2, p.412-8, 2000. TAKAHACHI, M.; TATSUGI, Y.; KOHNO, T. Endocrinal and pathological effects of anabolic-androgenic steroid in male rats. Endocrine Journal, Tokyo, v.51, p.425-34, 2004.

TRIFUNOVIC, B.; NORTON, G.R.; DUFFIELD, M.J.; AVRAAM, P.; WOODIWISS, A.J. An androgenic steroid decreases left ventricular compliance in rats. American Journal of Physiology: Heart and Circulatory Physiology, Bethesda, v.268, p. H1096-1105, 1995.

WATANABE, K.; TANAKA, H.; WEN, F.Q.; YOSHIDA, M. Effect of cytokines on thrombin-stimulated increases in intracellular calcium and PGI2 production by cultured human umbilical vein endothelial cells. Cellular Signalling, Oxford, v.8, p.247-51, 1996.

WATERS, R.E.; ROTEVATIN, S.; PING, L.; ANEXX, B.H.; YAN, Z. Voluntary running induces fiber type-specific angiogenesis in mouse skeletal muscle. American Journal of Physiology: Cell Physiology, Bethesda, v.287, p.C1342-8, 2004. YU-YAHIRO, J.A.; MICHAEL, R.H.; NASRALLAH, D.V.; SCHOFIELD, B. Morphological and histologic abnormalities in female and male rats treated with anabolic steroid. American Journal of Sports Medicine, Baltimore, v.17, p.686-9, 1989.

ENDEREÇO

Edilamar Menezes de Oliveira Departamento de Biodinâmica do Movimento do Corpo Humano Escola de Educação Física e Esporte - USP Av. Prof. Mello Moraes, 65 05508-030 - São Paulo - SP - BRASIL e-mail: edilamar@usp.br

Recebido para publicação: 20/10/2008

Revisado: 04/05/2009

Aceito: 02/06/2009 